\title{
Coincidence and Common Fixed Point of Weakly Compatible Maps in Fuzzy Metric Space
}

\author{
Saurabh Manro $^{1 *}$, Sumitra ${ }^{2}$ \\ ${ }^{1}$ School of Mathematics and Computer Applications, Thapar University, Patiala, India \\ ${ }^{2}$ Department of Mathematics, Faculty of Science, Jazan University, Jazan, KSA \\ Email: sauravmanro@yahoo.com, , sauravmanro@hotmail.com, mathsqueen d@yahoo.com
}

Received 24 February 2014; revised 24 March 2014; accepted 1 April 2014

Copyright (C) 2014 by authors and Scientific Research Publishing Inc.

This work is licensed under the Creative Commons Attribution International License (CC BY). http://creativecommons.org/licenses/by/4.0/

(c) (i) Open Access

\begin{abstract}
The aim of this paper is to establish some new common fixed point theorems for generalized contractive maps in fuzzy metric space by using property (E.A.), common property (E.A.), JCLR $R_{S T}$ property and $C L R_{s}$ property. Our results improve and extend the results of Chauhan et al. [1] and Sedghi et al. [2] besides several known results. We also furnish an illustrative example in support of our results.
\end{abstract}

\section{Keywords}

FM-Space, Weakly Compatible Maps, $C L R_{g}$ Property, Property (E.A.), The Common Property (E.A.), $J C L R_{S T}$ Property and Common Fixed Point

\section{Introduction}

Aamri et al. [3] generalized the concept of non compatibility by defining the notion of property (E.A.) and proved common fixed point theorems under strict contractive conditions. Many authors have proved common fixed point theorems in different settings for different contractive conditions. For details, we refer to [4]-[13].

In 2005, Liu et al. [14] further improved it by common property (E.A) while proving common fixed point theorems under strict contractive conditions. Recently, Sintunavarat et al. [13], defined the notion of $\left(C L R_{g}\right)$ property which is more general than (E.A) property.

Very recently, Manro et al. [15] introduced the notion of $\left(C L R_{S}\right)$ property and Chauhan et al. [4] introduced the notion of $\left(J C L R_{S T}\right)$ property.

*Corresponding author. 
The aim of this paper is to establish some new common fixed point theorems for generalized contractive maps in fuzzy metric space by using property (E.A.), common property (E.A.), $J C L R_{S T}$ property and $C L R_{S}$ property. Our results improve and extend the results of Chauhan et al. [1] and Sedghi et al. [2] besides several known results.

\section{Preliminaries}

Definition 2.1. [16] Let $X$ be any set. A fuzzy set in $X$ is a function with domain $X$ and values in [0,1].

The concept of triangular norms (t-norms) is originally introduced by Menger [17] in study of statistical metric spaces.

Definition 2.2. [18] A binary operation*: $[0,1] \times[0,1] \rightarrow[0,1]$ is continuous $t$-norm if * satisfies the following conditions:

i) * is commutative and associative;

ii) * is continuous;

iii) $a * 1=a$ for all $a \in[0,1]$;

iv) $a * b \leq c * d$ whenever $a \leq c$ and $b \leq d$ for all $a, b, c, d \in[0,1]$.

Examples of $t$-norms are:

$a * b=\min \{a, b\}, a * b=a b$ and $a * b=\max \{a+b-1,0\}$ for all $a, b \in[0,1]$.

Definition 2.3. [6] A 3-tuple $(X, M, *)$ is a fuzzy metric space if $X$ is an arbitrary set, * is a continuous $t$-norm and $M$ is a fuzzy set on $X^{2} \times[0, \infty)$ satisfying the following conditions for all $x, y, z \in X$ and $s, t>0$

i) $M(x, y, t)>0$;

ii) $M(x, y, t)=1$ for all $t>0$ if and only if $x=y$;

iii) $M(x, y, t)=M(y, x, t)$;

iv) $M(x, y, t) * M(y, z, s) \leq M(x, z, t+s)$;

v) $M(x, y,):.[0, \infty) \rightarrow[0,1]$ is continuous.

The function $M(x, y, t)$ denote the degree of nearness between $x$ and $y$ with respect to $t$.

In all that follows $(X, M, *)$ is a fuzzy metric space with the following property:

vi) $\lim _{t \rightarrow \infty} M(x, y, t)=1$ for all $x, y \in X$ and $t>0$.

Now we give some interesting examples of $F M$-spaces:

Example 2.1. Let $(X, d)$ be a metric space. Define $a * b=a+b$, for all $a, b \in[0,1] ; x, y \in X$ and $t>0$. Define $M(x, y, t)=t /(t+d(x, y))$. Then $(X, M, *)$ is a $F M$-space.

Moreover, fuzzy metric $M$ induced by a metric $d$ is often referred to as the Standard fuzzy metric.

Definition 2.4. [6]. A sequence $\left\{x_{n}\right\}$ in fuzzy metric space $(X, M, *)$ is

i) convergent to a point $x \in X$ if

$\lim _{n \rightarrow \infty} M\left(x_{n}, x, t\right)=1$ for all $t>0$,

ii) Cauchy sequence if

$\lim _{n \rightarrow \infty} M\left(x_{n+p}, x_{n}, t\right)=1$

for all $t>0$ and $p>0$.

Definition 2.5. A pair of self maps $(S, T)$ of a fuzzy metric space $(X, M, *)$ is

i) compatible [19] if

$\lim _{n \rightarrow \infty} M\left(S T x_{n}, T S x_{n}, t\right)=1$

for all $t>0$, whenever $\left\{x_{n}\right\}$ is a sequence in $X$ such that $\lim _{n \rightarrow \infty} S x_{n}=\lim _{n \rightarrow \infty} T x_{n}=z$ for some $z \in X$.

ii) non-compatible if there exists at least one sequence $\left\{x_{n}\right\}$ in $X$ such that $\lim _{n \rightarrow \infty} S x_{n}=\lim _{n \rightarrow \infty} T x_{n}=z$ for some $z \in X$ but either $\lim _{n \rightarrow \infty} M\left(S T x_{n}, T S x_{n}, t\right) \neq 1$ or non-existent.

iii) weakly compatible [20] if $S$ and $T$ commute at coincidence points, that is, $S T x=T S x$ whenever $S x=T x$.

iv) satisfy the property (E.A) [3] if there exist a sequence $\left\{x_{n}\right\}$ in $X$ such that $\lim _{n \rightarrow \infty} S x_{n}=\lim _{n \rightarrow \infty} T x_{n}=Z$ for some $z \in X$.

v) satisfies the common limit in the range of $T$ property $\left(C L R_{T}\right)$ [13] if there exist a sequence $\left\{x_{n}\right\}$ in $X$ such 
that $\lim _{n \rightarrow \infty} S x_{n}=\lim _{n \rightarrow \infty} T x_{n}=T z$ for some $z \in X$.

Definition 2.6. Two pairs of self maps $(A, S)$ and $(B, T)$ of fuzzy metric space $\left(X, M,^{*}\right)$ is

i) satisfy the common property (E.A) [14] if there exist two sequences $\left\{x_{n}\right\}$ and $\left\{y_{n}\right\}$ in $X$ such that $\lim _{n \rightarrow \infty} A x_{n}=\lim _{n \rightarrow \infty} S x_{n}=\lim _{n \rightarrow \infty} B y_{n}=\lim _{n \rightarrow \infty} T y_{n}=z$ for some $z \in X$.

ii) satisfy the (JCLR $\left.R_{S T}\right)$ property (with respect to maps $S$ and $\left.T\right)[4]$ if there exist two sequences $\left\{x_{n}\right\}$ and $\left\{y_{n}\right\}$ in $X$ such that

$\lim _{n \rightarrow \infty} A x_{n}=\lim _{n \rightarrow \infty} S x_{n}=\lim _{n \rightarrow \infty} B y_{n}=\lim _{n \rightarrow \infty} T y_{n}=S z=T z$ for some $z \in X$.

iii) satisfy the $\left(C L R_{S}\right)$ property (with respect to maps $\left.S\right)$ [15] if there exist two sequences $\left\{x_{n}\right\}$ and $\left\{y_{n}\right\}$ in $X$ such that

$\lim _{n \rightarrow \infty} A x_{n}=\lim _{n \rightarrow \infty} S x_{n}=\lim _{n \rightarrow \infty} B y_{n}=\lim _{n \rightarrow \infty} T y_{n}=S z$

for some $z \in X$.

Lemma 2.1. [7] If for all $x, y \in X, t>0$ and for a number $q \in(0,1), M(x, y, q t) \geq M(x, y, t)$ then $x=y$.

\section{Main Results}

Let $\Phi$ be the set of all increasing and continuous functions $\phi:(0,1] \rightarrow(0,1]$ such that $\phi(t)>t$ for all $t \in(0,1]$.

Example 3.1: Let $\phi:(0,1] \rightarrow(0,1]$ defined by $\phi(t)=\sqrt{t}$ for all $t \in(0,1]$. Clearly, $\phi \in \Phi$.

Theorem 3.1: Let $A, B, S$ and $T$ be self mappings of a fuzzy metric space $(X, M, *)$ satisfying the following:

$$
\begin{gathered}
M(A x, B y, t) \geq \phi\left(\operatorname { m i n } \left\{M(S x, T y, t), \sup _{t_{1}+t_{2}=\frac{2}{k} t} \min \left\{M\left(A x, S x, t_{1}\right), M\left(T y, B y, t_{2}\right)\right\},\right.\right. \\
\left.\left.\sup _{t_{3}+t_{4}=\frac{2}{k} t} \max \left\{M\left(A x, T y, t_{3}\right), M\left(B y, S x, t_{4}\right)\right\}\right\}\right)
\end{gathered}
$$

for all $x, y \in X, t>0$ and for some $1 \leq k<2, \phi \in \Phi$;

(3.2) $A(X) \subseteq T(X)$ and $B(X) \subseteq S(X)$;

(3.3) pair $(A, S)$ or $(B, T)$ satisfies the property (E.A);

(3.4) the range of one of the maps $A, B, S$ or $T$ is a closed subset of $X$.

Then pairs $(A, S)$ and $(B, T)$ have coincidence point. Further if $(A, S)$ and $(B, T)$ be weakly compatible pairs of self maps of fuzzy metric space $(X, M, *)$ then $A, B, S$ and $T$ have a unique common fixed point in $X$.

Proof: If the pair $(B, T)$ satisfies the property (E.A.), then there exist a sequence $\left\{x_{n}\right\}$ in $X$ such that $\lim _{n \rightarrow \infty} B x_{n}=\lim _{n \rightarrow \infty} T x_{n}=z$ for some $z \in X$.

Since, $B(X) \subseteq S(X)$, therefore, there exist a sequence $\left\{y_{n}\right\}$ in $X$ such that $B x_{n}=S y_{n}$. Hence, $\lim _{n \rightarrow \infty} S y_{n}=z$. Also, since $A(X) \subseteq T(X)$, there exist a sequence $\left\{z_{n}\right\}$ in $X$ such that $T x_{n}=A z_{n}$. Hence, $\lim _{n \rightarrow \infty} A z_{n}=z$.

Suppose that $S(X)$ is a closed subset of $X$. Then $z=S u$ for some $u \in X$. Therefore, $\lim _{n \rightarrow \infty} A z_{n}=\lim _{n \rightarrow \infty} S y_{n}=\lim _{n \rightarrow \infty} B x_{n}=\lim _{n \rightarrow \infty} T x_{n}=z=S u$.

We first claim that $A u=z$.

If $A u \neq z$, then there exist $t_{0}>0$ such that

$$
M\left(A u, z, \frac{2}{k} t_{0}\right)>M\left(A u, z, t_{0}\right) .
$$

The inequality (3.5) is always true when $A u \neq z$. To support our claim, we suppose on contrary that (3.5) is not true all $t>0$, i.e.,

$$
M\left(A u, z, \frac{2}{k} t\right)=M(A u, z, t) .
$$

Now, using equality (3.6) repeatedly, we get 


$$
M(A u, z, t)=M\left(A u, z, \frac{2}{k} t\right)=M\left(A u, z,\left(\frac{2}{k}\right)^{2} t\right)=\cdots=M\left(A u, z,\left(\frac{2}{k}\right)^{n} t\right) \rightarrow 1
$$

as $n \rightarrow \infty$. This gives, $M(A u, z, t)=1$ for all $t>0$. Hence, $A u=z$, which gives contradiction.

Therefore, inequality (3.5) is always true for some $t_{0}>0$.

Using (3.1), take $x=u, y=y_{n}$, we get

$$
\begin{gathered}
M\left(A u, B y_{n}, t_{0}\right) \geq \phi\left(\operatorname { m i n } \left\{M\left(S u, T y_{n}, t_{0}\right), \sup _{t_{1}+t_{2}=\frac{2}{k} t_{0}} \min \left\{M\left(A u, S u, t_{1}\right), M\left(T y_{n}, B y_{n}, t_{2}\right)\right\},\right.\right. \\
\left.\left.\sup _{t_{3}+t_{4}=\frac{2}{k} t_{0}} \max \left\{M\left(A u, T y_{n}, t_{3}\right), M\left(B y_{n}, S u, t_{4}\right)\right\}\right\}\right)
\end{gathered}
$$

let $t_{2}=t_{4}=\varepsilon$ then $t_{1}=t_{3}=\frac{2}{k} t_{0}-\varepsilon$ where $\varepsilon \in\left(0, \frac{2}{k} t_{0}\right)$ and $n \rightarrow \infty$, we get

$$
\begin{aligned}
& M\left(A u, z, t_{0}\right) \geq \phi\left(\operatorname { m i n } \left\{M\left(z, z, t_{0}\right), \min \left\{M\left(A u, z, \frac{2}{k} t_{0}-\varepsilon\right), M(z, z, \varepsilon)\right\},\right.\right. \\
& \left.\left.\quad \max \left\{M\left(A u, z, \frac{2}{k} t_{0}-\varepsilon\right), M(z, z, \varepsilon)\right\}\right\}\right) \\
& M\left(A u, z, t_{0}\right) \geq \phi\left(\min \left\{1, \min \left\{M\left(A u, z, \frac{2}{k} t_{0}-\varepsilon\right), 1\right\}, \max \left\{M\left(A u, z, \frac{2}{k} t_{0}-\varepsilon\right), 1\right\}\right\}\right) \\
& M\left(A u, z, t_{0}\right) \geq \phi\left(M\left(A u, z, \frac{2}{k} t_{0}-\varepsilon\right)\right)>M\left(A u, z, \frac{2}{k} t_{0}-\varepsilon\right) .
\end{aligned}
$$

As $\varepsilon \rightarrow 0$, we get

$$
M\left(A u, z, t_{0}\right) \geq M\left(A u, z, \frac{2}{k} t_{0}\right)
$$

which gives contradiction, hence

$$
A u=z .
$$

Therefore, $A u=z=S u$ which shows that $u$ is a coincidence point of the pair $(A, S)$. As $A$ and $S$ are weakly compatible. Therefore, $A S u=S A u$ and then $A A u=A S u=S A u=S S u$.

On the other hand, since $A(X) \subseteq T(X)$, there exist $v$ in $X$ such that $A u=T v$.

Now, we show that $B v=z$.

If $B v \neq z$, then again, as done above, there exist $t_{0}>0$ such that

$$
M\left(B v, z, \frac{2}{k} t_{0}\right)>M\left(B v, z, t_{0}\right) .
$$

The inequality (3.7) is always true when $B v \neq z$.

Using (3.1), take $x=u, y=v$, we have

$$
\begin{aligned}
M\left(A u, B v, t_{0}\right) \geq \phi( & \min \left\{M\left(S u, T v, t_{0}\right), \sup _{t_{1}+t_{2}=\frac{2}{k} t_{0}} \min \left\{M\left(A u, S u, t_{1}\right), M\left(T v, B v, t_{2}\right)\right\},\right. \\
& \left.\left.\sup _{t_{3}+t_{4}=\frac{2}{k} t_{0}} \max \left\{M\left(A u, T v, t_{3}\right), M\left(B v, S u, t_{4}\right)\right\}\right\}\right)
\end{aligned}
$$

let $t_{1}=t_{3}=\varepsilon$ then $t_{2}=t_{4}=\frac{2}{k} t_{0}-\varepsilon$ where $\varepsilon \in\left(0, \frac{2}{k} t_{0}\right)$, we get 


$$
\begin{aligned}
& M\left(z, B v, t_{0}\right) \geq \phi\left(\operatorname { m i n } \left\{M\left(z, z, t_{0}\right), \min \left\{M(z, z, \varepsilon), M\left(z, B v, \frac{2}{k} t_{0}-\varepsilon\right)\right\},\right.\right. \\
& \left.\left.\max \left\{M(z, z, \varepsilon), M\left(B v, z, \frac{2}{k} t_{0}-\varepsilon\right)\right\}\right\}\right) \\
& M\left(z, B v, t_{0}\right) \geq \phi\left(\min \left\{1, \min \left\{1, M\left(z, B v, \frac{2}{k} t_{0}-\varepsilon\right)\right\}, \max \left\{1, M\left(z, B v, \frac{2}{k} t_{0}-\varepsilon\right)\right\}\right\}\right) \\
& M\left(z, B v, t_{0}\right) \geq \phi\left(M\left(z, B v, \frac{2}{k} t_{0}-\varepsilon\right)\right)>M\left(z, B v, \frac{2}{k} t_{0}-\varepsilon\right) .
\end{aligned}
$$

As $\varepsilon \rightarrow 0$, we get

$$
M\left(z, B v, t_{0}\right) \geq M\left(z, B v, \frac{2}{k} t_{0}\right)
$$

which gives contradiction, hence $B v=z$.

Therefore, $B v=z=A u=T v$ which shows that $B v=T v$, i.e., $v$ is a coincidence point of the pair $(B, T)$. As $B$ and $T$ are weakly compatible, therefore, $B T v=T B v$ and hence, $B T v=T B v=T T v=B B v$.

Next, we show that $A A u=A u$, if not, then again as done above, there exist $t_{0}>0$ such that

$$
M\left(A A u, A u, \frac{2}{k} t_{0}\right)>M\left(A A u, A u, t_{0}\right) .
$$

Using (3.1), take $x=A u, y=v$, we have

$$
\begin{aligned}
M\left(A A u, B v, t_{0}\right) \geq \phi\left(\operatorname { m i n } \left\{M\left(S A u, T v, t_{0}\right), \sup _{t_{1}+t_{2}=\frac{2}{k} t_{0}} \min \left\{M\left(A A u, S A u, t_{1}\right), M\left(T v, B v, t_{2}\right)\right\},\right.\right. \\
\left.\sup _{t_{3}+t_{4}=\frac{2}{k} t_{0}} \max \left\{M\left(A A u, T v, t_{3}\right), M\left(B v, S A u, t_{4}\right)\right\}\right)
\end{aligned}
$$

let $t_{2}=t_{4}=\varepsilon$ then $t_{1}=t_{3}=\frac{2}{k} t_{0}-\varepsilon$ where $\varepsilon \in\left(0, \frac{2}{k} t_{0}\right)$, we get

$$
\begin{aligned}
& M\left(A A u, A u, t_{0}\right) \geq \phi\left(\operatorname { m i n } \left\{M\left(A A u, A u, t_{0}\right), \min \left\{M\left(A A u, A A u, \frac{2}{k} t_{0}-\varepsilon\right), M(z, z, \varepsilon)\right\},\right.\right. \\
& \left.\left.\quad \max \left\{M\left(A A u, A u, \frac{2}{k} t_{0}-\varepsilon\right), M(A u, A A u, \varepsilon)\right\}\right\}\right) \\
& M\left(A A u, A u, t_{0}\right) \geq \phi\left(\min \left\{M\left(A A u, A u, t_{0}\right), 1, \max \left\{M\left(A A u, A u, \frac{2}{k} t_{0}-\varepsilon\right), M(A A u, A u, \varepsilon)\right\}\right\}\right) \\
& M\left(A A u, A u, t_{0}\right) \geq \phi\left(M\left(A A u, A u, \frac{2}{k} t_{0}-\varepsilon\right)\right)>M\left(A A u, A u, \frac{2}{k} t_{0}-\varepsilon\right) .
\end{aligned}
$$

As $\varepsilon \rightarrow 0$, we get $M\left(A A u, A u, t_{0}\right) \geq M\left(A A u, A u, \frac{2}{k} t_{0}\right)$ which gives contradiction, hence $A A u=A u$.

Therefore, $A A u=A u=S A u$ and $A u$ are a common fixed point of $A$ and $S$. Similarly, we can prove that $B v$ is a common fixed point of $B$ and $T$. As $A u=B v$, we conclude that $A u$ is a common fixed point of $A, B, S$ and $T$.

The proof is similar when $T(X)$ is assumed to be a closed subset of $X$. The cases in which $A(X)$ or $B(X)$ is a closed subset of $X$ are similar to the cases in which $T(X)$ or $S(X)$ respectively, is closed since

$$
A(X) \subseteq T(X), \quad B(X) \subseteq S(X) .
$$


For uniqueness; let $w$ be another fixed point of $A, B, S$ and $T$. Then by (3.1), we have

$$
\begin{gathered}
M\left(A z, B w, t_{0}\right) \geq \phi\left(\operatorname { m i n } \left\{M\left(S z, T w, t_{0}\right), \sup _{t_{1}+t_{2}=\frac{2}{k} t_{0}} \min \left\{M\left(A z, S z, t_{1}\right), M\left(T w, B w, t_{2}\right)\right\},\right.\right. \\
\left.\left.\sup _{t_{3}+t_{4}=\frac{2}{k} t_{0}} \max \left\{M\left(A z, T w, t_{3}\right), M\left(B w, S z, t_{4}\right)\right\}\right\}\right) \\
M\left(z, w, t_{0}\right) \geq \phi\left(\operatorname { m i n } \left\{M\left(z, w, t_{0}\right), \sup _{t_{1}+t_{2}=\frac{2}{k} t_{0}} \min \left\{M\left(z, z, t_{1}\right), M\left(w, w, t_{2}\right)\right\},\right.\right. \\
\left.\left.\sup _{t_{3}+t_{4}=\frac{2}{k} t_{0}} \max \left\{M\left(z, w, t_{3}\right), M\left(w, z, t_{4}\right)\right\}\right\}\right)
\end{gathered}
$$

let $t_{1}=t_{3}=\varepsilon$ then $t_{2}=t_{4}=\frac{2}{k} t_{0}-\varepsilon$ where $\varepsilon \in\left(0, \frac{2}{k} t_{0}\right)$,

$$
\begin{gathered}
M\left(z, w, t_{0}\right) \geq \phi\left(\operatorname { m i n } \left\{M\left(z, w, t_{0}\right), \min \left\{M(z, z, \varepsilon), M\left(w, w, \frac{2}{k} t_{0}-\varepsilon\right)\right\},\right.\right. \\
\left.\left.\max \left\{M(z, w, \varepsilon), M\left(w, z, \frac{2}{k} t_{0}-\varepsilon\right)\right\}\right\}\right)
\end{gathered}
$$

as $\varepsilon \rightarrow 0$, we get

$$
\begin{aligned}
& M\left(z, w, t_{0}\right) \geq \phi\left(\min \left\{M\left(z, w, t_{0}\right), 1, M\left(w, z, \frac{2}{k} t_{0}\right)\right\}\right) \\
& M\left(z, w, t_{0}\right) \geq \phi\left(M\left(w, z, t_{0}\right)\right)>M\left(w, z, t_{0}\right)
\end{aligned}
$$

a contradiction, hence, $w=z$. It implies that $A, B, S$ and $T$ have unique common fixed point in $X$.

Hence the result.

Now we attempt to drop containment of subspaces by replacing property (E.A.) by a weaker condition common property (E.A.) in Theorem 3.1.

Theorem 3.2: Let $A, B, S$ and $T$ be self mappings of a fuzzy metric space $\left(X, M,{ }^{*}\right)$ satisfying condition (3.1) of Theorem 3.1 and the following:

(3.9) the pair $(A, S)$ and $(B, T)$ share the common (E.A.) property;

(3.10) $S(X)$ and $T(X)$ are closed subsets of $X$.

Then the pairs $(A, S)$ and $(B, T)$ have a point of coincidence each. Moreover, $A, B, S$ and $T$ have a unique common fixed point provided both the pairs $(A, S)$ and $(B, T)$ are weakly compatible.

Proof: In view of (3.2), there exist two sequences $\left\{x_{n}\right\}$ and $\left\{y_{n}\right\}$ in $X$ such that

$$
\lim _{n \rightarrow \infty} A x_{n}=\lim _{n \rightarrow \infty} S x_{n}=\lim _{n \rightarrow \infty} B y_{n}=\lim _{n \rightarrow \infty} T y_{n}=Z
$$

for some $z \in X$.

Since $S(X)$ is a closed subset of $X$, therefore, there exists a point $u$ in $X$ such that $z=S u$.

We claim that $A u=z$. If $A u \neq z$, then there exist $t_{0}>0$ such that

$$
M\left(A u, z, \frac{2}{k} t_{0}\right)>M\left(A u, z, t_{0}\right) .
$$

The inequality (3.11) is always true when $A u \neq z$. To support our claim, we suppose on contrary that (3.11) is not true all $t>0$, i.e.,

$$
M\left(A u, z, \frac{2}{k} t\right)=M(A u, z, t) .
$$

Now, using equality (3.12) repeatedly, we get 


$$
\begin{aligned}
M(A u, z, t) & =M\left(A u, z, \frac{2}{k} t\right)=M\left(A u, z,\left(\frac{2}{k}\right)^{2} t\right) \\
& =\cdots=M\left(A u, z,\left(\frac{2}{k}\right)^{n} t\right) \rightarrow 1
\end{aligned}
$$

as $n \rightarrow \infty$. This gives, $M(A u, z, t)=1$ for all $t>0$. Hence, $A u=z$, which gives contradiction.

Therefore, inequality (3.11) is always true for some $t_{0}>0$. Using (3.1), take $x=u, y=y_{n}$, we get

$$
\begin{aligned}
M\left(A u, B y_{n}, t_{0}\right) & \\
\geq \phi(\min & \left\{M\left(S u, T y_{n}, t_{0}\right), \sup _{t_{1}+t_{2}=\frac{2}{k} t_{0}} \min \left\{M\left(A u, S u, t_{1}\right), M\left(T y_{n}, B y_{n}, t_{2}\right)\right\},\right. \\
& \left.\left.\sup _{t_{3}+t_{4}=\frac{2}{k} t_{0}} \max \left\{M\left(A u, T y_{n}, t_{3}\right), M\left(B y_{n}, S u, t_{4}\right)\right\}\right\}\right)
\end{aligned}
$$

let $t_{2}=t_{4}=\varepsilon$ then $t_{1}=t_{3}=\frac{2}{k} t_{0}-\varepsilon$ where $\varepsilon \in\left(0, \frac{2}{k} t_{0}\right)$ and $n \rightarrow \infty$, we get

$$
\begin{aligned}
& M\left(A u, z, t_{0}\right) \geq \phi\left(\operatorname { m i n } \left\{M\left(z, z, t_{0}\right), \min \left\{M\left(A u, z, \frac{2}{k} t_{0}-\varepsilon\right), M(z, z, \varepsilon)\right\},\right.\right. \\
& \left.\left.\quad \max \left\{M\left(A u, z, \frac{2}{k} t_{0}-\varepsilon\right), M(z, z, \varepsilon)\right\}\right\}\right) \\
& M\left(A u, z, t_{0}\right) \geq \phi\left(\min \left\{1, \min \left\{M\left(A u, z, \frac{2}{k} t_{0}-\varepsilon\right), 1\right\}, \max \left\{M\left(A u, z, \frac{2}{k} t_{0}-\varepsilon\right), 1\right\}\right\}\right) \\
& M\left(A u, z, t_{0}\right) \geq \phi\left(M\left(A u, z, \frac{2}{k} t_{0}-\varepsilon\right)\right)>M\left(A u, z, \frac{2}{k} t_{0}-\varepsilon\right) .
\end{aligned}
$$

As $\varepsilon \rightarrow 0$, we get $M\left(A u, z, t_{0}\right) \geq M\left(A u, z, \frac{2}{k} t_{0}\right)$ which gives contradiction, hence $A u=z$.

Therefore, $A u=z=S u$ which shows that $u$ is a coincidence point of the pair $(A, S)$.

Since $T(X)$ is also a closed subset of $X$, therefore $\lim _{n \rightarrow \infty} T y_{n}=z$ in $T(X)$ and hence there exists $v$ in $X$ such that $T v=z=A u=S u$. Now, we show that $B v=z$.

If $B v \neq z$, then again as done above, there exist $t_{0}>0$ such that

$$
M\left(B v, z, \frac{2}{k} t_{0}\right)>M\left(B v, z, t_{0}\right) .
$$

The inequality (3.13) is always true when $B v \neq z$.

Using (3.1), take $x=u, y=v$, we have

$$
\begin{aligned}
& M\left(A u, B v, t_{0}\right) \\
& \geq \phi\left(\operatorname { m i n } \left\{M\left(S u, T v, t_{0}\right), \sup _{t_{1}+t_{2}=\frac{2}{k} t_{0}} \min \left\{M\left(A u, S u, t_{1}\right), M\left(T v, B v, t_{2}\right)\right\},\right.\right. \\
& \left.\left.\quad \sup _{t_{3}+t_{4}=\frac{2}{k} t_{0}} \max \left\{M\left(A u, T v, t_{3}\right), M\left(B v, S u, t_{4}\right)\right\}\right\}\right)
\end{aligned}
$$

let $t_{1}=t_{3}=\varepsilon$ then $t_{2}=t_{4}=\frac{2}{k} t_{0}-\varepsilon$ where $\varepsilon \in\left(0, \frac{2}{k} t_{0}\right)$, we get 


$$
\begin{aligned}
& M\left(z, B v, t_{0}\right) \geq \phi\left(\operatorname { m i n } \left\{M\left(z, z, t_{0}\right), \min \left\{M(z, z, \varepsilon), M\left(z, B v, \frac{2}{k} t_{0}-\varepsilon\right)\right\},\right.\right. \\
& \left.\left.\quad \max \left\{M(z, z, \varepsilon), M\left(B v, z, \frac{2}{k} t_{0}-\varepsilon\right)\right\}\right\}\right) \\
& M\left(z, B v, t_{0}\right) \geq \phi\left(\min \left\{1, \min \left\{1, M\left(z, B v, \frac{2}{k} t_{0}-\varepsilon\right)\right\}, \max \left\{1, M\left(z, B v, \frac{2}{k} t_{0}-\varepsilon\right)\right\}\right\}\right) \\
& M\left(z, B v, t_{0}\right) \geq \phi\left(M\left(z, B v, \frac{2}{k} t_{0}-\varepsilon\right)\right)>M\left(z, B v, \frac{2}{k} t_{0}-\varepsilon\right) .
\end{aligned}
$$

As $\varepsilon \rightarrow 0$, we get

$$
M\left(z, B v, t_{0}\right) \geq M\left(z, B v, \frac{2}{k} t_{0}\right)
$$

which gives contradiction, hence $B v=z$.

Therefore, $B v=z=T v$ which shows that $v$ is a coincidence point of the pair $(B, T)$.

Since the pairs $(A, S)$ and $(B, T)$ are weakly compatible and $A u=S u, B v=T v$, therefore, $A z=A S u=S A u=S z$, $B z=B T v=T B v=T z$.

If $A z \neq z$, then again as done above, there exist $t_{0}>0$ such that

$$
M\left(A z, z, \frac{2}{k} t_{0}\right)>M\left(A z, z, t_{0}\right) .
$$

Using (3.1), take $x=z, y=v$, we have

$$
\begin{aligned}
M\left(A z, B v, t_{0}\right) \geq \phi( & \min \left\{M\left(S z, T v, t_{0}\right), \sup _{t_{1}+t_{2}=\frac{2}{k} t_{0}} \min \left\{M\left(A z, S z, t_{1}\right), M\left(T v, B v, t_{2}\right)\right\},\right. \\
& \left.\left.\sup _{t_{3}+t_{4}=\frac{2}{k} t_{0}} \max \left\{M\left(A z, T v, t_{3}\right), M\left(B v, S z, t_{4}\right)\right\}\right\}\right)
\end{aligned}
$$

let $t_{2}=t_{4}=\varepsilon$ then $t_{1}=t_{3}=\frac{2}{k} t_{0}-\varepsilon$ where $\varepsilon \in\left(0, \frac{2}{k} t_{0}\right)$, we get

$$
\begin{aligned}
& M\left(A z, z, t_{0}\right) \geq \phi\left(\operatorname { m i n } \left\{M\left(z, z, t_{0}\right), \min \left\{M\left(A z, z, \frac{2}{k} t_{0}-\varepsilon\right), M(z, z, \varepsilon)\right\},\right.\right. \\
& \left.\left.\quad \max \left\{M\left(A z, z, \frac{2}{k} t_{0}-\varepsilon\right), M(z, z, \varepsilon)\right\}\right\}\right) \\
& M\left(A z, z, t_{0}\right) \geq \phi\left(\min \left\{1, \min \left\{M\left(A z, z, \frac{2}{k} t_{0}-\varepsilon\right), 1\right\}, \max \left\{M\left(A z, z, \frac{2}{k} t_{0}-\varepsilon\right), 1\right\}\right\}\right) \\
& M\left(A z, z, t_{0}\right) \geq \phi\left(M\left(A z, z, \frac{2}{k} t_{0}-\varepsilon\right)\right)>M\left(A z, z, \frac{2}{k} t_{0}-\varepsilon\right) .
\end{aligned}
$$

As $\varepsilon \rightarrow 0$, we get

$$
M\left(A z, z, t_{0}\right) \geq M\left(A z, z, \frac{2}{k} t_{0}\right)
$$

which gives contradiction, hence $A z=z$.

Therefore, $A z=z=S z$.

Similarly, one can prove that $B z=T z=z$. Hence, $A z=B z=S z=T z$, and $z$ is common fixed point of $A, B, S$ and $T$. 
Uniqueness easily follows by the use of inequality (3.1).

Hence the result.

Now we attempt to drop containment of subspaces by using weaker condition $J C L R_{S T}$ property in Theorem 3.2.

Theorem 3.3: Let $A, B, S$ and $T$ be four selfmaps in fuzzy metric space $(X, M, *)$ satisfying condition (3.1) of Theorem 3.1 and (3.15) $(A, S)$ and $(B, T)$ shares the $J C L R_{S T}$ property.

Then pairs $(A, S)$ and $(B, T)$ have coincidence point. Further if $(A, S)$ and $(B, T)$ be weakly compatible pair of self maps of $X$ then $A, B, S$ and $T$ have a unique common fixed point in $X$.

Proof: The pairs $(A, S)$ and $(B, T)$ satisfy the $\left(J C L R_{S T}\right)$ property, then there exist two sequences $\left\{x_{n}\right\}$ and $\left\{y_{n}\right\}$ in $X$ such that $\lim _{n \rightarrow \infty} A x_{n}=\lim _{n \rightarrow \infty} S x_{n}=\lim _{n \rightarrow \infty} B y_{n}=\lim _{n \rightarrow \infty} T y_{n}=S u=T u$ for some $u \in X$.

Firstly, we claim that $T u=B u$. Suppose not, then there exist $t_{0}>0$ such that

$$
M\left(T u, B u, \frac{2}{k} t_{0}\right)>M\left(T u, B u, t_{0}\right) .
$$

The inequality (3.16) is always true when $T u \neq B u$. To support our claim, we suppose on contrary that (3.16) is not true all $t>0$, i.e.,

$$
M\left(T u, B u, \frac{2}{k} t\right)=M(T u, B u, t)
$$

Now, using equality (3.17) repeatedly, we get

$$
M(T u, B u, t)=M\left(T u, B u, \frac{2}{k} t\right)=M\left(T u, B u,\left(\frac{2}{k}\right)^{2} t\right)=\cdots=M\left(T u, B u,\left(\frac{2}{k}\right)^{n} t\right) \rightarrow 1
$$

as $n \rightarrow \infty$. This gives, $M(T u, B u, t)=1$ for all $t>0$. Hence, $T u=B u$, which gives contradiction.

Therefore, inequality (3.16) is always true for some $t_{0}>0$.

Using (3.1), take $x=x_{n}, y=u$, we get

$$
\begin{gathered}
M\left(A x_{n}, B u, t_{0}\right) \geq \phi\left(\operatorname { m i n } \left\{M\left(S x_{n}, T u, t_{0}\right), \sup _{t_{1}+t_{2}=\frac{2}{k} t_{0}} \min \left\{M\left(A x_{n}, S x_{n}, t_{1}\right), M\left(T u, B u, t_{2}\right)\right\},\right.\right. \\
\left.\left.\sup _{t_{3}+t_{4}=\frac{2}{k} t_{0}} \max \left\{M\left(A x_{n}, T u, t_{3}\right), M\left(B u, S x_{n}, t_{4}\right)\right\}\right\}\right)
\end{gathered}
$$

let $t_{1}=t_{3}=\varepsilon$ then $t_{2}=t_{4}=\frac{2}{k} t_{0}-\varepsilon$ where $\varepsilon \in\left(0, \frac{2}{k} t_{0}\right)$ and $n \rightarrow \infty$, we get

$$
\begin{gathered}
M\left(T u, B u, t_{0}\right) \geq \phi\left(\operatorname { m i n } \left\{M\left(T u, T u, t_{0}\right), \min \left\{M(T u, T u, \varepsilon), M\left(T u, B u, \frac{2}{k} t_{0}-\varepsilon\right)\right\},\right.\right. \\
\left.\left.\max \left\{M(T u, T u, \varepsilon), M\left(B u, T u, \frac{2}{k} t_{0}-\varepsilon\right)\right\}\right\}\right) \\
M\left(T u, B u, t_{0}\right) \geq \phi\left(\min \left\{1, \min \left\{1, M\left(T u, B u, \frac{2}{k} t_{0}-\varepsilon\right)\right\}, \max \left\{1, M\left(T u, B u, \frac{2}{k} t_{0}-\varepsilon\right)\right\}\right\}\right) \\
M\left(T u, B u, t_{0}\right) \geq \phi\left(M\left(T u, B u, \frac{2}{k} t_{0}-\varepsilon\right)\right)>M\left(T u, B u, \frac{2}{k} t_{0}-\varepsilon\right)
\end{gathered}
$$

As $\varepsilon \rightarrow 0$, we get

$$
M\left(T u, B u, t_{0}\right) \geq M\left(T u, B u, \frac{2}{k} t_{0}\right)
$$

which gives contradiction, hence $T u=B u$. 
Next, we show that $A u=T u$. Suppose not, then again as done above, there exist $t_{0}>0$ such that

$$
M\left(A u, T u, \frac{2}{k} t_{0}\right)>M\left(A u, T u, t_{0}\right) .
$$

Using (3.1), take $x=u, y=y_{n}$, we get

$$
\begin{aligned}
& M\left(A u, B y_{n}, t_{0}\right) \\
& \geq \phi\left(\operatorname { m i n } \left\{M\left(S u, T y_{n}, t_{0}\right), \sup _{t_{1}+t_{2}=\frac{2}{k} t_{0}} \min \left\{M\left(A u, S u, t_{1}\right), M\left(T y_{n}, B y_{n}, t_{2}\right)\right\},\right.\right. \\
& \left.\left.\quad \sup _{t_{3}+t_{4}=\frac{2}{k} t_{0}} \max \left\{M\left(A u, T y_{n}, t_{3}\right), M\left(B y_{n}, S u, t_{4}\right)\right\}\right\}\right)
\end{aligned}
$$

let $t_{2}=t_{4}=\varepsilon$ then $t_{1}=t_{3}=\frac{2}{k} t_{0}-\varepsilon$ where $\varepsilon \in\left(0, \frac{2}{k} t_{0}\right)$ and $n \rightarrow \infty$, we get

$$
\begin{gathered}
M\left(A u, T u, t_{0}\right) \geq \phi\left(\min \left\{M\left(S u, S u, t_{0}\right), \min \left\{M\left(A u, T u, \frac{2}{k} t_{0}-\varepsilon\right), M(T u, T u, \varepsilon)\right\},\right)\right. \\
\left.\left.\max \left\{M\left(A u, T u, \frac{2}{k} t_{0}-\varepsilon\right), M(T u, T u, \varepsilon)\right\}\right\}\right) \\
M\left(A u, T u, t_{0}\right) \geq \phi\left(\min \left\{1, \min \left\{M\left(A u, T u, \frac{2}{k} t_{0}-\varepsilon\right), 1\right\}, \max \left\{M\left(A u, T u, \frac{2}{k} t_{0}-\varepsilon\right), 1\right\}\right\}\right) \\
M\left(A u, T u, t_{0}\right) \geq \phi\left(M\left(A u, T u, \frac{2}{k} t_{0}-\varepsilon\right)\right)>M\left(A u, T u, \frac{2}{k} t_{0}-\varepsilon\right)
\end{gathered}
$$

As $\varepsilon \rightarrow 0$, we get $M\left(A u, T u, t_{0}\right) \geq M\left(A u, T u, \frac{2}{k} t_{0}\right)$ which gives contradiction, hence $A u=T u$. Hence, $A u$ $=B u=S u=T u=z$ (say). Since the pair $(A, S)$ is weakly compatible, $A S u=S A u$ and then $A z=S z$. Similarly, as the pair $(B, T)$ is weakly compatible, $B T u=T B u$ and then $T z=B z$.

Next, we claim that $A z=z$, suppose not. Then by (3.1), take $x=z, y=u$, we get

$$
\begin{aligned}
& M\left(A z, B u, t_{0}\right) \\
& \geq \phi\left(\operatorname { m i n } \left\{M\left(S z, T u, t_{0}\right), \sup _{t_{1}+t_{2}=\frac{2}{k} t_{0}} \min \left\{M\left(A z, S z, t_{1}\right), M\left(T u, B u, t_{2}\right)\right\},\right.\right. \\
& \left.\left.\quad \sup _{t_{3}+t_{4}=\frac{2}{k} t_{0}} \max \left\{M\left(A z, T u, t_{3}\right), M\left(B u, S z, t_{4}\right)\right\}\right\}\right)
\end{aligned}
$$

let $t_{1}=t_{3}=\varepsilon$ then $t_{2}=t_{4}=\frac{2}{k} t_{0}-\varepsilon$ where $\varepsilon \in\left(0, \frac{2}{k} t_{0}\right)$,

$$
\begin{gathered}
M\left(A z, z, t_{0}\right) \geq \phi\left(\operatorname { m i n } \left\{M\left(A z, z, t_{0}\right), \min \left\{M(A z, A z, \varepsilon), M\left(z, z, \frac{2}{k} t_{0}-\varepsilon\right)\right\},\right.\right. \\
\left.\left.\max \left\{M(A z, z, \varepsilon), M\left(z, A z, \frac{2}{k} t_{0}-\varepsilon\right)\right\}\right\}\right) \\
M\left(A z, z, t_{0}\right) \geq \phi\left(\min \left\{M\left(A z, z, t_{0}\right), 1, \max \left\{M(A z, z, \varepsilon), M\left(z, A z, \frac{2}{k} t_{0}-\varepsilon\right)\right\}\right\}\right)
\end{gathered}
$$

As $\varepsilon \rightarrow 0$, we get 


$$
\begin{aligned}
& M\left(A z, z, t_{0}\right) \geq \phi\left(\min \left\{M\left(A z, z, t_{0}\right), 1, \max \left\{M(A z, z, 0), M\left(z, A z, \frac{2}{k} t_{0}\right)\right\}\right\}\right) \\
& M\left(A z, z, t_{0}\right) \geq \phi\left(\min \left\{M\left(A z, z, t_{0}\right), 1, M\left(z, A z, \frac{2}{k} t_{0}\right)\right\}\right) \\
& M\left(A z, z, t_{0}\right) \geq \phi\left(M\left(z, A z, t_{0}\right)\right)>M\left(z, A z, t_{0}\right)
\end{aligned}
$$

a contradiction, hence, $A z=B z=z$. Therefore, $z$ is a common fixed point of $A$ and $B$. Similarly, we prove that $S z$ $=T z=z$ by taking $x=u, y=z$ in (3.1). Therefore, we conclude that $z=A z=B z=S z=T z$ this implies that $A, B$, $S$ and $T$ have common fixed point in $X$.

Uniqueness easily follows by the use of inequality (3.1).

Next we attempt to drop closedness of range of maps and relax containment of two subspaces to one subspace by replacing property (E.A.) by a weaker condition $C L R_{S}$ property in Theorem 3.1.

Theorem 3.4: Let $A, B, S$ and $T$ be four selfmaps fuzzy metric space $(X, M, *)$ satisfying condition (3.1) of Theorem 3.1 and

(3.19) $(A, S)$ and $(B, T)$ shares the $C L R_{S}$ property $\left(C L R_{T}\right.$ property)

(3.20) $A(X) \subset T(X) \quad(B(X) \subset S(X))$.

Then pairs $(A, S)$ and $(B, T)$ have coincidence point. Further if $(A, S)$ and $(B, T)$ be weakly compatible pair of self maps of $X$ then $A, B, S$ and $T$ have a unique common fixed point in $X$.

Proof: Proof of this theorem easily follows on same lines of Theorem 3.2.

On taking $A=B$ and $S=T$ in Theorem 3.1 then we get the following interesting result which is improved version of Theorem 1 of Sedghi et al. [2].

Corollary 3.1: Let $A$ and $S$ be self mappings of a fuzzy metric space $(X, M, *)$ satisfying the following:

$$
\begin{gathered}
M(A x, A y, t) \geq \phi\left(\operatorname { m i n } \left\{M(S x, S y, t), \sup _{t_{1}+t_{2}=\frac{2}{k} t} \min \left\{M\left(A x, S x, t_{1}\right), M\left(S y, A y, t_{2}\right)\right\},\right.\right. \\
\left.\left.\sup _{t_{3}+t_{4}=\frac{2}{k} t} \max \left\{M\left(A x, S y, t_{3}\right), M\left(A y, S x, t_{4}\right)\right\}\right\}\right)
\end{gathered}
$$

for all $x, y \in X, t>0$ and for some $1 \leq k<2, \phi \in \Phi$;

(3.22) $A(X) \subseteq S(X)$;

(3.23) pair $(A, S)$ satisfies the property (E.A)

(3.24) $A(X)$ or $S(X)$ is a closed subset of $X$.

Then pair $(A, S)$ has a coincidence point. Further, if pair $A$ and $S$ be weakly compatible self maps of fuzzy metric space $(X, M, *)$, then $A$ and $S$ have a unique common fixed point in $X$.

On taking $A=B$ and $S=T$ in Theorem 3.4 then we get the following interesting result which is improved version of Theorem 3.3 of Chauhan et al. [3].

Corollary 3.2: Let $A$ and $S$ be self mappings of a fuzzy metric space $(X, M, *)$ satisfying the following:

(3.25) $(A, S)$ satisfies the $C L R_{S}$ property.

Then pair $(A, S)$ has a coincidence point. Further if pair $A$ and $S$ be weakly compatible self maps of $X$ then $A$ and $S$ has a unique common fixed point in $X$.

Finally, we conclude this paper by furnishing example to demonstrate Theorem 3.3 besides exhibiting its superiority over earlier relevant results.

Example 3.2. Let $\left(X, M,^{*}\right)$ be a fuzzy metric space where $a * b=a$. $b$ for all $a, b \in[0,1]$ and $X=[3,19)$. Let $\phi:(0,1] \rightarrow(0,1]$ be defined as $\phi(t)=\sqrt{t}$ for all $t \in(0,1]$, Clearly, $\phi \in \Phi$. Define $A, B, S$ and $T$ by

$$
A(x)=\left\{\begin{array}{ll}
1 & x \in\{1\} \cup(3,15) \\
x+11 & x \in(1,3]
\end{array}, B(x)=\left\{\begin{array}{ll}
1 & x \in\{1\} \cup(3,15) \\
x+5 & x \in(1,3]
\end{array},\right.\right.
$$




$$
S(x)= \begin{cases}1 & x=1 \\ 6 & x \in(1,3] \\ \frac{x+1}{4} & x \in(3,15)\end{cases}
$$

and

$$
T(x)= \begin{cases}1 & x=1 \\ 11 & x \in(1,3] . \\ x-2 & x \in(3,15)\end{cases}
$$

Take $\left\{x_{n}\right\}=\left\{y_{n}\right\}=\left\{3+\frac{1}{n}\right\}$, clearly

$$
\lim _{n \rightarrow \infty} A x_{n}=\lim _{n \rightarrow \infty} S x_{n}=\lim _{n \rightarrow \infty} B y_{n}=\lim _{n \rightarrow \infty} T y_{n}=1=T 1=S 1
$$

for some $1 \in X$.

Thus, $(A, S)$ and $(B, T)$ satisfies $J C L R_{S T}$ property.

Also, $A X=\{1\} \cup(12,14], \quad B X=\{1\} \cup(6,8], \quad S X=[1,4) \cup\{6\}, T X=(1,13)$ and condition (3.1) is satisfied by maps $A, B, S$ and $T$. Thus, the maps $A, B, S$ and $T$ satisfy all conditions of Theorem 3.3. Hence, $A, B, S$ and $T$ have a unique common fixed point $x=1$.Moreover it should be noted that $A X, B X, S X$ and $T X$ are not closed subsets of $X$. Also, $A X \not \subset T X$ and $B X \not \subset S X$. Also, $A, B, S$ and $T$ are all discontinuous maps at fixed point $x=$ 1.

Definition 3.1 [21] Two families of self mappings $\left\{A_{i}\right\}_{i=1}^{m}$ and $\left\{B_{j}\right\}_{j=1}^{n}$ are said to be pairwise commuting if

i) $A_{i} A_{j}=A_{j} A_{i}, i, j \in\{1,2,3, \cdots, m\}$,

ii) $B_{i} B_{j}=B_{j} B_{i}, i, j \in\{1,2,3, \cdots, n\}$,

iii) $A_{i} B_{j}=B_{j} A_{i}, \quad i \in\{1,2,3, \cdots, n\}, j \in\{1,2,3, \cdots, n\}$.

As an application of Theorem 3.2, we prove a common fixed point theorem for four finite families of mappings on fuzzy metric spaces. While proving our result, we utilize Definition 3.1 which is a natural extension of commutativity condition to two finite families.

Theorem 3.4: Let $\left\{A_{1}, A_{2}, \cdots, A_{m}\right\},\left\{B_{1}, B_{2}, \cdots, B_{n}\right\},\left\{S_{1}, S_{2}, \cdots, S_{p}\right\}$ and $\left\{T_{1}, T_{2}, \cdots, T_{q}\right\}$ be four finite families of self mappings of a fuzzy metric space $(X, M, *)$ such that $A=A_{1} \cdot A_{2} \cdots A_{m}, B=B_{1} \cdot B_{2} \cdots B_{n}$, $S=S_{1} \cdot S_{2} \cdots S_{p}$ and $T=T_{1} \cdot T_{2} \cdots T_{q}$ satisfying the conditions (3.1), (3.9), (3.10) and (3.26) the pairs of families $\left(\left\{A_{i}\right\},\left\{S_{k}\right\}\right)$ and $\left(\left\{B_{r}\right\},\left\{T_{t}\right\}\right)$ commute pairwise.

Then the pairs $(A, S)$ and $(B, T)$ have a point of coincidence each. Moreover, $\left\{A_{i}\right\}_{i=1}^{m},\left\{S_{k}\right\}_{k=1}^{p},\left\{B_{r}\right\}_{r=1}^{n}$ and $\left\{T_{t}\right\}_{t=1}^{q}$ have a unique common fixed point.

Proof: By using (3.26), we first show that $A S=S A$ as

$$
\begin{aligned}
A S & =\left(A_{1} A_{2} \cdots A_{m}\right)\left(S_{1} S_{2} \cdots S_{p}\right)=\left(A_{1} A_{2} \cdots A_{m-1}\right)\left(A_{m} S_{1} S_{2} \cdots S_{p}\right)=\left(A_{1} A_{2} \cdots A_{m-1}\right)\left(S_{1} S_{2} \cdots S_{p} A_{m}\right) \\
& =\left(A_{1} A_{2} \cdots A_{m-2}\right)\left(A_{m-1} S_{1} S_{2} \cdots S_{p} A_{m}\right)=\left(A_{1} A_{2} \cdots A_{m-2}\right)\left(S_{1} S_{2} \cdots S_{p} A_{m-1} A_{m}\right) \\
& =\cdots=A_{1}\left(S_{1} S_{2} \cdots S_{p} A_{2} \cdots A_{m}\right)=\left(S_{1} S_{2} \cdots S_{p}\right)\left(A_{1} A_{2} \cdots A_{m}\right)=S A
\end{aligned}
$$

Similarly one can prove that $B T=T B$. And hence, obviously the pair $(A, S)$ is compatible and $(B, T)$ is weakly compatible. Now using Theorem 3.1, we conclude that $A, S, B$ and $T$ have a unique common fixed point in $X$, say $z$.

Now, one needs to prove that $z$ remains the fixed point of all the component mappings.

For this consider

$$
\begin{aligned}
A\left(A_{i} z\right) & =\left(\left(A_{1} A_{2} \cdots A_{m}\right) A_{i}\right) z=\left(A_{1} A_{2} \cdots A_{m-1}\right)\left(A_{m} A_{i}\right) z=\left(A_{1} A_{2} \cdots A_{m-1}\right)\left(A_{i} A_{m}\right) z \\
& =\left(A_{1} A_{2} \cdots A_{m-2}\right)\left(A_{m-1} A_{i} A_{m}\right) z=\left(A_{1} A_{2} \cdots A_{m-2}\right)\left(A_{i} A_{m-1} A_{m}\right) z \\
& =\cdots=A_{1}\left(A_{i} A_{2} \cdots A_{m}\right) z=\left(A_{1} A_{i}\right)\left(A_{2} \cdots A_{m}\right) z \\
& =\left(A_{i} A_{1}\right)\left(A_{2} \cdots A_{m}\right) z=A_{i}\left(A_{1} A_{2} \cdots A_{m}\right) z=A_{i} A z=A_{i} z
\end{aligned}
$$


Similarly, one can prove that

$$
\begin{gathered}
A\left(S_{k} z\right)=S_{k}(A z)=S_{k} z, \\
S\left(S_{k} z\right)=S_{k}(S z)=S_{k} z, \\
S\left(A_{i} z\right)=A_{i}(S z)=A_{i} z, \\
B\left(B_{r} z\right)=B_{r}(B z)=B_{r} z, \\
B\left(T_{t} z\right)=T_{t}(B z)=T_{t} z, \\
T\left(T_{t} z\right)=T_{t}(T z)=T_{t} z
\end{gathered}
$$

and

$$
T\left(B_{r} z\right)=B_{r}(T z)=B_{r} z,
$$

which show that (for all $i, r, k$ and $t$ ) $A_{i} z$ and $S_{k} z$ are other fixed point of the pair $(A, S)$ whereas $B_{r} z$ and $T_{t} z$ are other fixed points of the pair $(B, T)$. As $A, B, S$ and $T$ have a unique common fixed point, so, we get

$$
z=A_{i} z=S_{k} z=B_{r} z=T_{t} z,
$$

for all $i=1,2, \cdots, m, \quad k=1,2, \cdots, p, \quad r=1,2, \cdots, n, \quad t=1,2, \cdots, q$. which shows that $z$ is a unique common fixed point of $\left\{A_{i}\right\}_{i=1}^{m},\left\{S_{k}\right\}_{k=1}^{p},\left\{B_{r}\right\}_{r=1}^{n}$ and $\left\{T_{t}\right\}_{t=1}^{q}$.

Remark 3.2: Theorem 3.4 is a slight but partial generalization of Theorem 3.2 as the commutativity requirements in this theorem are slightly stronger as compared to Theorem 3.1.

Remark 3.3. From the above results, it is asserted that for the existence of common fixed point of two pairs of self maps in fuzzy metric spaces satisfying $J C L R_{S T}$ property the following conditions are never required:
a) the containment of ranges amongst the involved maps;
b) the completeness of the whole space/subspace;
c) the closedness of space/subspaces;
d) continuity requirement amongst the involved maps.

\section{Acknowledgements}

The first author wishes to thank Dr. B. E. Rhoades, Dr. Sanjay Kumar and Dr. S. S. Bhatia and the referees for their very helpful suggestions and many kind comments. The second author wishes to thank DSR, Jizan University, Saudi Arabia for financial support for the publication of this research.

\section{References}

[1] Chauhan, S., Bhatnagar, S. and Radenovic, S. (2013) Common Fixed Point Theorem for Weakly Compatible Mappings in Fuzzy Metric Spaces. Le Matematiiche, 68, 87-98.

[2] Sedghi, S., Shobe, N. and Aliouche, A. (2010) A Common Fixed Point Theorem for Weakly Compatible Mappings in Fuzzy Metric Spaces. General Mathematics, 18, 3-12.

[3] Aamri, M. and El Moutawakil, D. (2002) Some New Common Fixed Point Theorems under Strict Contractive Conditions. Journal of Mathematical Analysis and Applications, 270, 181-188. http://dx.doi.org/10.1016/S0022-247X(02)00059-8

[4] Chauhan, S., Sintunavarat, W. and Kumam, P. (2012) Common Fixed Point Theorems for Weakly Compatible Mappings in Fuzzy Metric Spaces Using (JCLR) Property. Applied Mathematics, 3, 976-982. http://dx.doi.org/10.4236/am.2012.39145

[5] Fang, J.X. and Gao, Y. (2009) Common Fixed Point Theorems under Strict Contractive Conditions in Menger Spaces. Nonlinear Analysis, 70, 184-193. http://dx.doi.org/10.1016/j.na.2007.11.045

[6] George, A. and Veeramani, P. (1994) On Some Results in Fuzzy Metric Spaces. Fuzzy Sets and Systems, 64, 395-399. http://dx.doi.org/10.1016/0165-0114(94)90162-7

[7] Grabiec, M. (1988) Fixed Points in Fuzzy Metric Spaces. Fuzzy Sets and Systems, 27, 385-389. http://dx.doi.org/10.1016/0165-0114(88)90064-4

[8] Gregori, V., Morillas, S. and Sapena, A. (2011) Examples of Fuzzy Metrics and Applications. Fuzzy Sets and Systems, 
170, 95-111. http://dx.doi.org/10.1016/j.fss.2010.10.019

[9] Kramosil, I. and Michalek, J. (1975) Fuzzy Metric and Statistical Spaces. Kybernetica, 11, 336-344.

[10] Kumar, S. and Fisher, B. (2010) A Common Fixed Point Theorem in Fuzzy Metric Space Using Property (E. A.) and Implicit Relation. Thai Journal of Mathematics, 8, 439-446.

[11] Mihet, D. (2010) Fixed Point Theorems in Fuzzy Metric Spaces Using Property E.A. Nonlinear Analysis, 73, 21842188. http://dx.doi.org/10.1016/j.na.2010.05.044

[12] Shostak, A.P. (1989) Two Decades of Fuzzy Topology; Basic Ideas, Notions and Results. Russian Math Surveys, 44, 123-186. http://dx.doi.org/10.1070/RM1989v044n06ABEH002295

[13] Sintunavarat, W. and Kumam, P. (2011) Common Fixed Point Theorems for a Pair of Weakly Compatible Mappings in Fuzzy Metric Spaces. Journal of Applied Mathematics, 2011, Article ID: 637958.

[14] Liu, W., Wu, J. and Li, Z. (2005) Common Fixed Points of Single-Valued and Multivalued Maps. International Journal of Mathematics and Mathematical Sciences, 19, 3045-3055. http://dx.doi.org/10.1155/IJMMS.2005.3045

[15] Manro, S., Kumar, S. and Bhatia, S.S. Common Fixed Point Theorems in Fuzzy Metric Spaces Using Common Range Property. Journal of Advanced Research in Applied Mathematics.

[16] Zadeh, L.A. (1965) Fuzzy Sets. Information and Control, 8, 338-353. http://dx.doi.org/10.1016/S0019-9958(65)90241-X

[17] Menger, K. (1942) Statistical Metrics. Proceedings of the National Academy of Sciences (USA), 28, 535-537. http://dx.doi.org/10.1073/pnas.28.12.535

[18] Schweizer, B. and Sklar, A. (1983) Probabilistic Metric Spaces. Amsterdam.

[19] Jungck, G. (1986) Compatible Mappings and Common Fixed Points. International Journal of Mathematics and Mathematical Sciences, 9, 771-779. http://dx.doi.org/10.1155/S0161171286000935

[20] Jungck, G. and Rhoades, B.E. (1998) Fixed Points for Set Valued Functions without Continuity. Indian Journal of Pure and Applied Mathematics, 29, 227-238.

[21] Imdad, M., Tanveer, M. and Hasan, M. (2010) Some Common Fixed Point Theorems in Menger PM-Spaces. Fixed Point Theory and Applications, 2010, Article ID: 819269. 